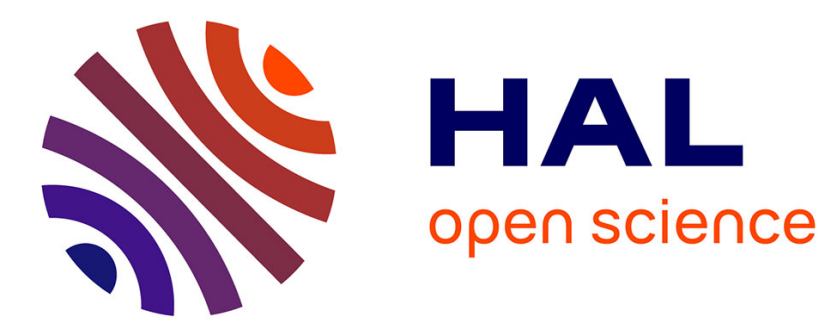

\title{
Les bases de la génétique quantitative. Définition et mesure des paramètres du croisement
}

\author{
Jean- Michel J.- . M. Brun
}

\section{To cite this version:}

Jean- Michel J.- . M. Brun. Les bases de la génétique quantitative. Définition et mesure des paramètres du croisement. Productions Animales, 1992, 1992, pp.101-105. hal-02712612

\section{HAL Id: hal-02712612 \\ https://hal.inrae.fr/hal-02712612}

Submitted on 1 Jun 2020

HAL is a multi-disciplinary open access archive for the deposit and dissemination of scientific research documents, whether they are published or not. The documents may come from teaching and research institutions in France or abroad, or from public or private research centers.
L'archive ouverte pluridisciplinaire HAL, est destinée au dépôt et à la diffusion de documents scientifiques de niveau recherche, publiés ou non, émanant des établissements d'enseignement et de recherche français ou étrangers, des laboratoires publics ou privés. 


\section{J.-M. BRUN}

INRA Station d'Amélioration Génétique des Animaux BP 2731326 Castanet-Tolosan Cedex
Les bases de la génétique quantitative

\section{Définition et mesure des paramètres du croisement}

Résumé. Comment décrire la variabilité génétique qui existe entre les valeurs moyennes des différents types génétiques issus d'un plan de croisement entre plusieurs populations ? Comment prédire la valeur moyenne des nombreux types génétiques que l'on peut obtenir, en fonction du système de croisement pratiqué, à partir de $n$ populations parentales disponibles ? Ces questions illustrent les deux grandes fonctions des paramètres du croisement : l'analyse génétique et l'amélioration génétique. Contrairement aux paramètres qui décrivent la variabilité génétique intra-population des variances, les paramètres du croisement sont des effets fixés, caractérisant les populations parentales étudiées. La première partie de l'article est consacrée à la modélisation du croisement. Nous présentons et analysons les "ingrédients" communs à de nombreux modèles du croisement : (1) les notions de "composante individuelle (ou directe)" et de "composante maternelle" (voire grand-maternelle) de la valeur moyenne d'un croisement, (2) les notions "d'effets moyens (ou additifs) des races parentales" et "d'effets d'hétérosis", (3) les composantes de l'hétérosis respectivement liées à la "dominance" et à "l'épistasie".

L'hétérosis, ou "vigueur hybride", fait l'objet d'un développement particulier concernant ses facteurs de variation, ses mécanismes et leurs conséquences pour la prédiction de l'hétérosis à partir du degré d'hétérozygotie. On présente les paramètres du modèle de Dickerson (1969), qui est le plus utilisé en génétique animale : les effets additifs respectivement direct, maternel et grand-maternel $\left(g^{\circ}, g^{m}, g^{n}\right)$, les effets d'hétérosis liés à la dominance respectivement direct et maternel $\left(h^{\circ}\right.$ et $\left.h^{m}\right)$ et les pertes épistatiques par recombinaison $\left(r^{\circ}\right.$ et $\left.r^{\prime \prime}\right)$. La deuxième partie de l'article est consacrée aux protocoles d'estimation des paramètres de Dickerson. Enfin un exemple d'estimation illustre à la fois l'application du modèle de Dickerson à un dispositif diallèle (tous les croisements possibles entre $n$ races prises $2 \grave{a}$ 2) et une méthode simple d'estimation des paramètres du croisement.

Dans les articles précédents on s'est intéressé à l'analyse de la variabilité génétique qui existe entre individus à l'intérieur d'une population. En amélioration génétique, ces modèles constituent la base théorique de la sélection intra-population. On s'intéresse maintenant au croisement entre diverses populations et à la variation génétique qui existe entre les valeurs moyennes de différents types génétiques issus d'un plan de croisement entre ces populations. Les paramètres du croisement sont les paramètres de cette variabilité.

L'analyse d'un plan de croisement correspond à deux grands types d'objectifs :

- L'analyse génétique : au même titre que la transplantation embryonnaire ou la pratique d'adoptions, le croisement permet une disjonction entre le génotype de la mère et celui du produit : lors d'un accouplement en race pure (mâle AA x femelle AA), le couple mère/produit a pour génotype $\mathrm{AA} / \mathrm{AA}$ alors que dans le croisement (mâle $\mathrm{BB} \times$ femelle $\mathrm{AA}$ ), il a pour génotype AA/BA. Le croisement constitue ainsi un outil pour évaluer la part respective de l'influence maternelle et de l'effet lié aux gènes du produit dans la détermination d'un caractère de ce produit.
- L'amélioration génétique : il s'agit alors de prédire la valeur zootechnique de différentes combinaisons possibles entre les populations parentales disponibles. C'est la base théorique du croisement.

\section{1 / Modélisation du croisement}

Il existe différents modèles du croisement. On peut distinguer des modèles plus "théoriques" construits sur les principes de la génétique quantitative (dont les paramètres sont définis d'emblée en termes d'effets des gènes) et des modèles plus "empiriques" pour lesquels on a dû a posteriori rechercher la signification génétique des paramètres. On peut également distinguer des modèles contingents de plans de croisement à structure particulière comme par exemple les plans qu'on appelle "diallèles" (ce sont des croisements factoriels entre $n$ populations utilisées comme "mâles" et les mêmes populations utilisées comme "femelles") et des modèles plus généraux, applicables à tout type de plan de croisement. Enfin, il existe une grande variété de modèles en fonction des phénomènes biologiques que l'on prend en compte. 
Après avoir exposé les "ingrédients" communs à de nombreux modèles, nous présenterons le modèle de Dickerson $(1969,1973)$ qui est le plus couramment utilisé en génétique animale.

\section{1 / Fondements "biologiques"}

\section{a / Effets directs et maternels}

Chez les animaux et en particulier chez les mammifêres, les contributions génétiques du père et de la mère sont inégales : en plus des gènes que la mère transmet à ses descendants, au même titre que le père, pour la réalisation d'un caractère, la mère peut influencer ce caractère à travers l'environnement qu'elle procure à ses descendants durant la gestation et l'allaitement. Cette influence peut avoir une base génétique chez la mère (gènes de production laitière de la mère, influençant la croissance du produit, par exemple).

La valeur phénotypique $P_{x}$ de l'individu $X$ de mère W, peut s'écrire :

$$
P_{x}=G_{x}^{0}+G_{w}^{m}+E
$$

où $G_{x}^{\circ}$ représente l'effet des gènes de l'individu sur le caractère considéré, qualifiés d'effets génétiques individuels ou encore directs. (L'indice "o", initiale de "offspring", est relatif à l'individu) ;

$\mathrm{G}_{\mathrm{w}}^{\mathrm{m}}$ représente l'effet des gènes de la mère, qualifiés d'effets génétiques maternels

et $\mathrm{E}$ un effet du milieu, autre que le milieu "maternel".

La somme $G_{x}^{o}+G_{w}^{m}$ représente la valeur génotypique globale de l'individu $\mathrm{X}$.

A son tour, l'influence maternelle peut être conditionnée par des effets maternels, ce qui constitue les effets grand-maternels. Les effets maternels ne sont bien sûr pas spécifiques du croisement mais le croisement les met particulièrement bien en évidence. Par exemple, dans le croisement entre les deux races de chevaux Shire Horse et Poney Shetland, le poids à la naissance des poulains de mère Shire Horse est trois fois plus élevé que celui des croisés réciproques et cette différence persiste jusqu'à l'âge adulte.

La valeur génotypique moyenne des deux croisements réciproques $\mathrm{AB}=$ mâle $\mathrm{A} \times$ femelle $\mathrm{B}$ et $\mathrm{BA}=$ mâle $\mathrm{B} x$ femelle A peut s'écrire :

$$
\left.\begin{array}{l}
G_{A B}=G_{A B}^{\circ}+G_{B}^{m} \\
G_{B A}=G_{A B}^{\circ}+G_{A}^{m}
\end{array}\right\} G_{A B}-G_{B A}=G_{B}^{m}-G_{A}^{m}
$$

La différence $G_{A B}-G_{B A}$ traduit ainsi les différences d'effets maternels entre les races $B$ et $A$.

La pratique du croisement entre une race qui contribue favorablement à $G^{\prime}$ utilisée comme "père" et une race à $G^{\mathrm{m}}$ favorable, donnant les mères, constitue un exemple d'utilisation de la complémentarité entre races.

\section{b / Additivité et hétérosis}

L'hétérosis, encore appelé "vigueur hybride" est un phénomène spécifique du croisement. L'hétérosis se définit comme la différence entre la performance moyenne du croisement de première génération entre deux populations $\bar{F}_{1}$ et la performance moyenne des populations parentales $\overline{\mathrm{P}}: \mathbf{H}_{1}=\overline{\mathbf{F}}_{1}-\overline{\mathbf{P}}$
L'hétérosis mesure ainsi l'écart par rapport à l'additivité des valeurs des parents au sens où la valeur du croisé ne résulte pas simplement d'une somme d'effets transmis par les parents.

Par extension, on parle aussi d'hétérosis dans divers croisements dérivés de la $F_{1}$ en termes de "fraction de l'hétérosis exprimée" (ou "retenue") dans ces croisements (par exemple, "hétérosis exprimée dans la deuxième génération de croisements ou $\mathrm{F}_{2}$ ”).

L'hétérosis est fonction du caractère considéré, des populations en présence mais aussi de l'environnement :

- En règle générale, l'hétérosis varie inversement à l'héritabilité du caractère. Les caractères liés à la reproduction (fertilité, prolificité, viabilité des jeunes) à faible héritabilité, montrent en général un effet d'hétérosis important, de l'ordre de 10 à $20 \%$. Les caractères liés au développement des jeunes (vitesse de croissance, par exemple) montrent des effets d'hétérosis plus faibles, de 5 à $8 \%$. Enfin, les caractères liés à la composition corporelle (caractéristiques de la carcasse, qualité de la viande), très héritables, ne manifestent pratiquement pas d'hétérosis.

- L'hétérosis est d'autant plus important que les populations parentales sont génétiquement plus éloignées. Un exemple en est donné dans l'article de Bidanel.

- Enfin l'hétérosis s'exprimerait plus en milieu "pauvre" qu'en milieu "riche", ce qui est lié à la plus grande stabilité des croisés face aux variations du milieu.

Les mécanismes de l'hétérosis sont complexes et encore mal élucidés. Le modèle génétique le plus classique qui en rend compte est le modèle de la dominance. Nous allons l'illustrer en prenant le cas d'un caractère à déterminisme génétique simple, supposé déterminé par 1 locus à 2 allèles $(\mathrm{A} / \mathrm{a})$. Soient 2 populations parentales $P_{1}$ et $P_{2}$, dans lesquelles les fréquences alléliques à ce locus sont respectivement $(\mathrm{p}$, $q$ ) et $\left(p^{\prime}, q^{\prime}\right)$. Les valeurs génotypiques sont a pour $A A$, $\mathrm{d}$ pour $\mathrm{Aa}$ et -a pour aa. La différence $d$ entre la valeur de l'hétérozygote et la moyenne des homozygotes est appelé "l'écart de dominance". Soit y la différence entre la fréquence des gènes dans les deux populations ( $\mathrm{y}^{2}$ mesure la distance génétique entre $P_{1}$ et $\left.P_{2}\right): y=p-p^{\prime}=q^{\prime}-q$.

Les valeurs génotypiques moyennes $\bar{P}_{1}, \overline{\mathrm{P}}_{2}$ et $\overline{\mathrm{F}}_{1}$ des deux populations parentales et de la population croisée F1 s'écrivent :

$$
\begin{aligned}
& \overline{\mathrm{P}}_{1}=\mathrm{a}(\mathrm{p}-\mathrm{q})+2 \mathrm{~d} p \mathrm{q} \\
& \overline{\mathrm{P}}_{2}=\mathrm{a}(\mathrm{p}-\mathrm{q}-2 \mathrm{y})+\mathrm{d}(\mathrm{pq}+\mathrm{y}(\mathrm{p}-\mathrm{q}-\mathrm{y})) \\
& \overline{\mathrm{F}}_{1}=\mathrm{a}(\mathrm{p}-\mathrm{q}-\mathrm{y})+\mathrm{d}(2 \mathrm{p} \mathrm{q}+\mathrm{y}(\mathrm{p}-\mathrm{q})) \\
& \text { L'hétérosis } \mathrm{H}_{1}=\overline{\mathrm{F}}_{1}-\overline{\mathrm{P}}_{\text {s'écrit alors : }} \\
& \qquad \mathrm{H}_{1}=\mathrm{d} \mathrm{y}^{2}
\end{aligned}
$$

L'hétérosis est ainsi une fonction directe de l'écart de dominance (d) et du carré des différences alléliques entre les deux populations $\left(\mathrm{y}^{2}\right)$. La formule (1) permet de comprendre comment l'hétérosis est fonction du caractère - à travers $d$ - et de la distance génétique entre les populations parentales - à travers $\mathrm{y}^{2}$.

D'autre part on peut montrer que $\mathrm{y}^{2}$ représente l'augmentation de la fréquence des hétérozygotes 
dans la population $F_{1}$ par rapport à leur fréquence moyenne dans les populations parentales. L'hétérosis découle ainsi de l'avantage de l'hétérozygotie (exprimé par d) et de l'accroissement de la fréquence des hétérozygotes (exprimé par $\mathrm{y}^{2}$ ) dans la population $F_{1}$.

L'existence d'hétérosis repose sur l'hypothèse d'une valeur positive de d c'est-à-dire une valeur de l'hétérozygote $\mathrm{Aa}$ ) plus proche de celle de l'homozygote favorable (AA) que de celle de l'homozygote défavorable. Une théorie des chaînes de réactions enzymatiques accrédite cette hypothèse : dans une chaîne métabolique, la liaison entre le flux de métabolites et la quantité (ou l'activité) d'un enzyme particulier de cette chaîne n'est pas linéaire mais hyperbolique. Cela signifie que pour un enzyme donné, impliqué dans une longue chaîne métabolique, une variation de quantité ou d'activité n'aura que très peu d'effet sur le flux. Par conséquent, l'hétérozygote Âa aura en général un effet physiologique proche de celui de AA.

Quand on considère plusieurs locus dont les effets s'ajoutent, c'est-à-dire en l'absence d'épistasie, l'hétérosis sera encore fonction des écarts de dominance et de la distance entre populations à chaque locus. En présence d'épistasie, l'hétérosis devient fonction de ce type d'interactions. Il peut ainsi y avoir de l'hétérosis en l'absence de dominance.

\section{c / Relation entre hétérosis et degré d'hétérozygotie}

On peut montrer qu'en présence de dominance seule, l'hétérosis est proportionnel au "degré d'hétérozygotie raciale" (dh), défini comme la probabilité que deux gènes homologues d'un individu croisé ne proviennent pas de la même race parentale. L'hétérosis est maximal dans le croisement de première génération où $\mathrm{dh}=1$. Dans la génération $\mathrm{F}_{2}$ issue du croisement d'animaux $F_{1}$ entre eux, $d h=0,5$ et l'hétérosis est diminué de moitié. Le niveau d'hétérosis est ensuite constant dans les générations successives $F_{3}$, $F_{4}$, etc. Dans les croisements en retour $P_{1} \times F_{1}$ et $P_{2} \times$ $F_{1}$ l'hétérosis attendu est le même qu'en $F_{2}$. Une expérience chez la souris montre cette proportionnalité entre l'hétérosis sur la taille de portée et le degré d'hétérozygotie de la femelle dans divers croisements réciproques de deux souches. Cependant, cette proportionnalité n'est pas toujours vérifiée. Par exemple, dans plusieurs expériences de croisements indépendantes entre deux races de poules, l'hétérosis sur la ponte observée en $F_{2}$ est systématiquement inférieur à la moitié de celui observé en $\mathrm{F}_{1}$ (4\% en moyenne contre $16 \%$ ). Dans d'autres expériences, l'écart concerne la moyenne de la $F_{2}$ qui s'avère inférieure à la moyenne des deux back-cross $\mathrm{P}_{1} \times \mathrm{F}_{1}$ et $\mathrm{P}_{2} \times \mathrm{F}_{1}$ malgré l'égalité de leur constitution génétique et de leur degré d'hétérozygotie.

Divers modèles génétiques qui introduisent l'épistasie permettent de rendre compte de ces phénomènes : Dickerson (1969, 1973), par exemple, fait l'hypothèse que la sélection a développé dans les populations parentales des associations favorables de gènes qui sont détruites par la recombinaison lorsque des individus croisés se reproduisent. Il introduit un paramètre " $r$ " appelé "pertes épistatiques dues à la recombinaison", dont l'expression est maximale en $\mathrm{F}_{2}$.

A côté de modèles particuliers comportant, comme celui de Dickerson, des hypothèses sur la nature de l'épistasie, des modèles génétiques généraux ont été proposés (Kinghorn 1980, Hill 1982). Tout en se limitant aux interactions pouvant exister entre deux locus seulement, ces modèles font intervenir un plus grand nombre de paramètres.

\section{d / Autres facteurs}

Certains auteurs introduisent d'autres facteurs dans leur modèle du croisement comme par exemple les effets liés au sexe ou encore les effets maternels d'origine cytoplasmique qu'ils distinguent de l'ensemble des effets maternels.

\section{2 / Le modèle de Dickerson $(1969,1973)$}

Les paramètres de ce modèle se rattachent aux trois composantes génétiques classiques de la décomposition de Fisher qui sont les effets additifs, de dominance et d'épistasie :

- g, valeur génétique "additive" ou encore "moyenne" d'une race, exprimée en écart à la moyenne de l'ensemble des races pures étudiés.

- $h$, appelé à tort "hétérosis", représente en fait la fraction de l'hétérosis lié à la dominance.

\section{- r, pertes épistasiques dues à la recombinaison.}

Cette décomposition s'applique à la composante directe de la valeur d'un croisement, liée aux gènes des individus, mais aussi à la composante maternelle et grand-maternelle de cette valeur. Les effets relatifs à la composante directe sont affectés de l'indice "o", comme "offspring" (ou parfois "I" comme "Individual") $: \mathrm{g}^{\circ}, \mathrm{h}^{\circ}, \mathrm{r}^{\circ}$. Ceux relatifs à la composante maternelle sont : $\mathrm{g}^{\mathrm{m}}, \mathrm{h}^{\mathrm{m}}$, $\mathrm{r}^{\mathrm{m}}$.et ceux relatifs à la composante grand-maternelle sont affectés de l'indice $n$.

Quelles sont les lois du modèle?

- Les effets additifs d'une race s'expriment proportionnellement à la contribution de cette race aux gènes de l'individu considéré

- Les effets d'hétérosis entre deux races s'expriment proportionnellement aux degrés d'hétérozygotie raciale de l'individu considéré pour ce couple de races. Par exemple, l'hétérosis direct dans le croisement A x BC s'écrira $\mathrm{H}^{\circ}=0,5 \mathrm{~h}_{\mathrm{AB}}^{\circ}+0,5 \mathrm{~h}_{\mathrm{AC}}^{\circ}$. Les effets d'hétérosis dans d'autres croisements sont donnés dans l'article de Bidanel au tableau 1.

- Les effets "r" ont un coefficient égal au pourcentage de paires de gènes dans les gamètes des deux parents qui sont les combinaisons non parentales. Ce paramètre exprime l'écart à la liaison linéaire entre hétérosis et degré d'hétérozygotie qui découle de l'existence des effets d'épistasie.

Un exemple d'application de ce modèle est donné au paragraphe 2.2 .

\section{2 / Estimation des paramètres du modèle de Dickerson}

\section{$2.1 /$ Protocole}

Il faut produire au moins autant de types génétiques qu'il y a de paramètres à estimer. Le choix de ces types est fonction des paramètres que l'on veut estimer. Le modèle génétique sert de guide pour le choix de ces types génétiques. 


\section{a / Estimation des valeurs additives directes et maternelles et de l'hétérosis direct : $\boldsymbol{g}^{\boldsymbol{o}}, \boldsymbol{g}^{\boldsymbol{m}}, \boldsymbol{h}^{\circ}$}

Un dispositif couramment utilisé est le diallèle, qui consiste en un plan de croisement factoriel entre n populations utilisées comme mâles et comme femelles. Le diallèle complet comporte $n^{2}$ croisements incluant $n$ génotypes purs et $n(n-1) / 2$ couples de croisements réciproques. Les croisements réciproques permettent l'estimation des effets maternels. Ce dispositif ne permet pas de dissocier les effets maternels et grand-maternels. Une estimation non biaisée des effets maternels, globalement calculés comme différence entre l'effet d'une race utilisée comme mère et comme père, suppose l'égalité du niveau génétique des mâles et des femelles d'une population. Un exemple d'estimation de ces paramètres est donné plus loin.

Classiquement, ces plans sont interprétés selon d'autres modèles que celui de Dickerson. Les paramètres de ces modèles sont les AGC (Aptitudes Générales à la Combinaison), les ASC (Aptitudes Spécifiques à la Combinaison), les effets maternels et les "effets de réciprocité spécifique". L’AGC d'une population est la valeur moyenne de sa descendance obtenue en croisement avec toutes les autres populations du dispositif diallèle. L'ASC d'un croisement particulier est l'écart entre la valeur de ce croisement et la somme des AGC de ses parents. Les effets de réciprocité spécifique s'interprètent comme des interactions entre AGC et effets maternels.

\section{b / Estimation de l'hétérosis maternel, séparation des effets maternels et grand-maternels : $\boldsymbol{h}^{m}$, $g^{n}$}

L'option la plus fréquemment choisie est un dispositif utilisant des femelles issues d'un diallèle en croisement avec des mâles d'une autre population. L'utilisation de croisements en retour permet également l'estimation de l'hétérosis maternel. Cependant les estimées de $\mathrm{h}^{\mathrm{m}}$ dans ces dispositifs sont biaisées par des effets d'épistasie. Le recours à des mâles croisés permet de lever ces biais.

\section{c / Estimation des paramètres relatifs à l'épistasie : $\boldsymbol{r}^{\boldsymbol{m}}, \boldsymbol{r}^{\mathrm{m}}$}

L'estimation des effets épistatiques de recombinaison nécessite le contrôle de la deuxième génération( $\mathrm{F}_{2}$ et croisements en retour avec deux races) pour les effets directs et de la troisième génération pour les effets maternels.

\section{2 / Exemple d'estimation des paramètres du croisement}

On analyse le poids à 11 semaines dans un plan de croisement diallèle entre deux souches de lapin, $\mathrm{A}$ et B. Ces deux souches ont été constituées en 1975 à partir d'une même souche d'origine. La souche $B$ est sélectionnée sur la taille de portée. La souche A n'est pas sélectionnée. Le tableau suivant donne les valeurs moyennes du poids à 11 semaines (en $\mathrm{g}$ ) pour les 4 types génétiques. L'écart-type de ces moyennes est de $20 \mathrm{~g}$.

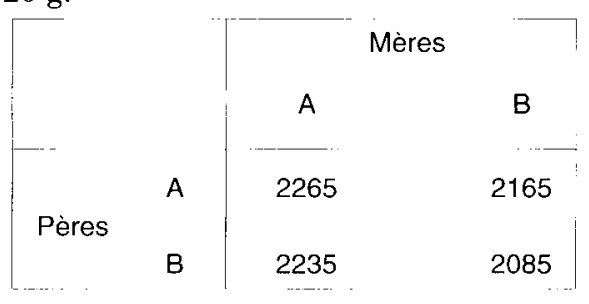

La décomposition de ces valeurs moyennes selon le modèle de Dickerson conduit au système d'équations suivant:

$$
\begin{aligned}
A A=\mu+g_{A}^{\circ}+\left(g_{A}^{m}+g_{A}^{n}\right) \\
\text { Mâle A x Femelle B= } A B=\mu+0,5\left(g_{A}^{o}+g_{B}^{o}\right)+h_{A B}^{o}+\left(g_{B}^{m}+g_{B}^{n}\right) \\
\text { Mâle B X Femelle } A=B A=\mu+0,5\left(g_{A}^{o}+g_{B}^{o}\right)+h_{A B}^{o}+\left(g_{A}^{m}+g_{A}^{n}\right) \\
B B=\mu+g_{B}^{o}+\left(g_{B}^{m}+g_{B}^{n}\right)
\end{aligned}
$$

Ce dispositif de croisement ne permet pas de séparer les effets maternels et grand-maternels ; on estimera leur somme $\mathrm{g}^{\mathrm{m}^{\prime}}=\mathrm{g}^{\mathrm{m}}+\mathrm{g}^{\mathrm{n}}$. Il reste 6 paramètres à estimer :

$$
\mu, g_{A}^{o}, g_{B}^{o}, h_{A B}^{o}, g_{A}^{m^{\prime}} \text { et } g_{B}^{m^{\prime}}
$$

Les 4 relations entre eux permettent d'en estimer 4. On pose :

$$
g_{A}^{0}+g_{B}^{0}=0 \text { et } g_{A}^{m^{\prime}} \text { et } g_{B}^{m^{\prime}}=0
$$

d'où 4 inconnues. La résolution du système conduit à :

$$
\begin{aligned}
& \mu=0,5(A A+B B)=2175 g \pm 15 g \\
& g_{A}^{o}=-g_{B}^{\circ}=0,5(A A-B B+A B-B A)=55 g \pm 20 g \\
& g_{A}^{m^{\prime}}=g_{B}^{m^{\prime}}=0,5(B A-A B)=35 g \pm 15 g \\
& h_{A B}^{\circ}=0,5(A B+B A-A A-B B)=25 g \pm 20 g
\end{aligned}
$$

Les effets $g^{\prime \prime}$ et $g^{m}$ sont significativement différents de 0 . La sélection réalisée dans la souche $B$ s'est accompagnée d'une réduction de $180 \mathrm{~g}$ du poids des lapereaux à 11 semaines. Cette réduction est attribuable aux effets directs des gènes $(2 \times 55=110 \mathrm{~g}$ ), mais aussi aux effets maternels ( $70 \mathrm{~g}$ ), vraisemblablement liés à l'augmentation de la taille de portée dans la souche $\mathrm{B}(+1$ lapereau). Cet exemple illustre l'intérêt du croisement pour l'analyse génétique.

\section{Conclusion}

Les modèles du croisement couramment utilisés sont généralement simples et sans doute peu réalistes : la description des interactions entre locus est simplifiée ; les interactions noyau x cytoplasme ne sont pas prises en compte comme composante de l'hétérosis; on néglige les interactions entre effets directs et maternels. Ces limites doivent être gardées à l'esprit lorsqu'on utilisera ces modèles pour le choix d'un système de croisement. 


\section{Références bibliographiques}

Cunningham E.P., 1987. Crossbreeding : The Greek temple model. J. Anim. Breed. Genet., 104, 2 - 11.

Dickerson G.E., 1969. Experimental approaches in utilizing breed ressources. Anim. Breed. Abstr., 37, 191-202.

Dickerson G.E., 1973. Inbreeding and heterosis in animals. In : Proc. Animal Breeding Symposium in Honour of Dr. Jay Lush, ASA, ADSA, Champaign, 54 - 77.

Eisen E.J., 1989. Genetic models to predict crossbred performance : a review. Rev. Brasil. Genet., 12, 13 - 26.

Foulley J.L., LefortG., 1978. Méthodes d'estimation des effets directs et maternels en sélection animale. Ann. Génét. Sél. Anim., 10, 475-496.
Hill W.G., 1982. Dominance and epistasis as components of heterosis. Z. Tierzüchtg. Züchtgsbiol., 99, 161 - 168.

Kinghorn B.P., 1980. The expression of recombination loss in quantitative traits. Z. Tierzüchtg. Züchtgsbiol,, 97, 138 143.

Sellier P., 1982. Selecting populations for use in crossbreeding. In Proc. 2nd World Congress on Genetics Applied to livestock Production, 4 - 8 October 1982, Madrid, Vol. II. 15 - 49.

Sölkner J., James J.W., 1990. Optimum Design of crossbreeding experiments. III. Efficiency of design without all purebreeds. J. Anim. Breed. Genet., 107, 421 - 430. 